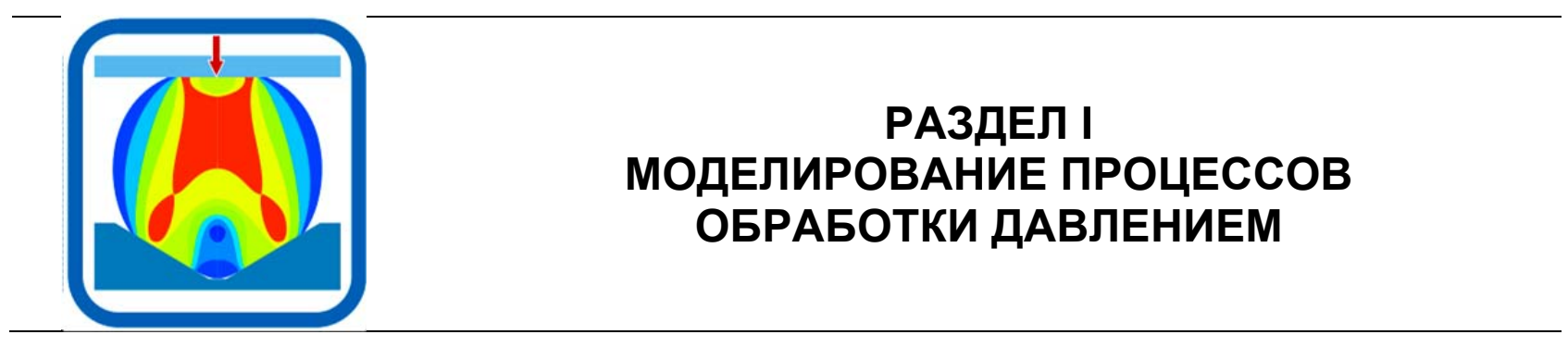

УДК 531.2

Чигиринский В. В. Науменко Е. Г.

\title{
НЕКОТОРЫЕ ОСОБЕННОСТИ РЕШЕНИЯ ПЛОСКОЙ ЗАДАЧИ МЕХАНИКИ СПЛОШНОЙ СРЕДЫ
}

На практике часто приходится рассматривать задачи механики сплошной среды, в которых одновременно присутствует упругая и пластическая деформация материала. Игнорирование одной из них приводит к неверным решением прикладных задач. К примеру, такие сочетания приводят к таким нежелательным явлениям, как ползучесть, усталость. Действительно, малая пластическая деформация, не учитываемая законом Гука, с течением времени увеличивается, приводя к перераспределению напряжений и деформаций. Эти свойства, называемые последействием и релаксацией, имеют общее название ползучесть [1]. Имеет место изменение напряженного состояния с течением времени, вследствие большого числа циклов периодически меняющейся во времени нагрузки. Такое проявление пластичности называется усталостью [2]. Многие вопросы прочности машин и сооружений опираются на выводы теории пластичности. Считается, что возникновение пластических деформаций в локальной зоне конструкции еще не означает потери ее несущей способности в целом [3]. Этот прогрессивный метод расчета широко используется при проектировании экономичных машин, сооружений и позволяет в большей степени использовать ресурс прочности (носит название несущая способность). Таких примеров на практике множество.

Однако интерес для технологов представляет случай, когда в очаге деформации в процессах обработки металлов давлением деформируемая среда находится в двух состояниях одновременно, упругом и пластичном [4]. Это случай присутствия зоны прилипания или зоны заторможенной пластической деформации. Действительно, контактные силы трения препятствуют перемещению металла на границе с инструментом. Их влияние распространяется на определенную глубину, занимая целую область очага деформации. Исследования показали [5], что остаточные линейные и сдвиговые деформации в данной области очага деформации отсутствуют. Следовательно, имеет место сочетание упругой и пластической деформации. Представляет практический и теоретический интерес определение соотношений указанных зон, их влияние на силовые и кинематические параметры процесса. Для этого возникает необходимость в получении соответствующих друг другу решений задач теории пластичности и упругости. В работах [6-10] предложен новый подход решения задач теории пластичности, связанный с использованием гармонических функций, или, так называемый, метод аргумент функций. Как показал анализ, в рамках этого метода появилась возможность получить решения для упругого и пластичного состояний материала, и сравнить полученные результаты.

Целью работы является обобщение результата целого комплекса практичных задач, которые позволяют расширить возможности решения с точки зрения граничных и начальных условий. Представляется целесообразным получить определенные обобщения решаемых плоских задач теории упругости и пластичности, таким образом, чтобы выявить условия существования решений в виде дифференциальных соотношений, накладываемых на разный конечный результат. 
Для решения плоской задачи теории пластичности использовалась следующая постановка задачи:

$$
\begin{gathered}
\frac{\partial \sigma_{x}}{\partial x}+\frac{\partial \tau_{x y}}{\partial y}=0 ; \quad \frac{\partial \tau_{y x}}{\partial x}+\frac{\partial \sigma_{y}}{\partial y}=0 ; \quad\left(\sigma_{x}-\sigma_{y}\right)^{2}+4 \cdot \tau_{x y}^{2}=4 \cdot k^{2} ; \quad \frac{\sigma_{x}-\sigma_{y}}{2 \cdot \tau_{x y}}=\frac{\xi_{x}-\xi_{y}}{\gamma_{x y}^{\prime}}=F_{1} ; \\
\xi_{x}+\xi_{y}=0 ; \frac{\partial^{2} \xi_{x}}{\partial y^{2}}+\frac{\partial^{2} \xi_{y}}{\partial x^{2}}=\frac{\partial^{2} \gamma_{x y}^{\prime}}{\partial y \partial x},
\end{gathered}
$$

где $\sigma_{x}$ - нормальное напряжение;

$\tau_{x y}-$ касательное напряжение;

$k$ - сопротивление пластической деформации сдвига;

$\xi_{x}, \xi_{y}, \gamma_{x y}^{\prime}-$ линейные и сдвиговая скорости деформаций.

Решением замкнутой задачи является, в напряжениях:

$$
\begin{gathered}
\sigma_{x}=C_{\sigma} \cdot \exp \theta^{\prime \prime} \cdot \cos A \Phi+\sigma_{0}+f(y)+C, \\
\sigma_{y}=-C_{\sigma} \cdot \exp \theta^{\prime \prime} \cdot \cos A \Phi+\sigma_{0}+f(x)+C, \\
\tau_{x y}=C_{\sigma} \cdot \exp \theta^{\prime \prime} \cdot \sin A \Phi .
\end{gathered}
$$

при

$$
\begin{gathered}
\theta_{x}^{\prime \prime}=\mp A \Phi_{y}, \quad \theta_{y}^{\prime \prime}= \pm A \Phi_{x}, \\
\theta_{x x}^{\prime \prime \prime}+\theta_{y y}^{\prime \prime \prime}=0, \quad A \Phi_{x x}+A \Phi_{y y}=0,
\end{gathered}
$$

в скоростях деформаций:

$$
\xi_{x}=-\xi_{y}=C_{\xi} \cdot \exp \theta^{\prime \prime \prime} \cdot \cos B_{1} \Phi, \quad \gamma_{x y}^{\prime}=2 \cdot C_{\xi} \cdot \exp \theta^{\prime \prime \prime} \cdot \sin B_{1} \Phi
$$

при

$$
\begin{gathered}
\theta_{x}^{\prime \prime \prime}=\mp \hat{A}_{1} \hat{O}_{y}, \quad \theta_{y}^{\prime \prime \prime}= \pm \hat{A}_{1} \hat{O}_{x}, \\
\theta_{x x}^{\prime \prime \prime}+\theta_{y y}^{\prime \prime \prime}=0, \quad B_{1} \Phi_{x x}+B_{2} \Phi_{y y}=0 .
\end{gathered}
$$

В выражениях (1), (3) присутствуют тригонометрические, экспоненциальные базовые функции и аргумент функции $A \Phi, \theta$, на которые наложены ограничения (2), (4) в виде соотношений Коши-Римана и уравнений Лапласа. Необходимо подчеркнуть, что решение задачи теории пластичности получено с помощью гармонических функций, которые нашли широкое применение в теории упругости.

Имеем классическую постановку плоской задачи в теории упругости:

$$
\begin{gathered}
\frac{\partial \sigma_{\mathrm{x}}}{\partial \mathrm{x}}+\frac{\partial \tau_{\mathrm{xy}}}{\partial \mathrm{y}}=0, \quad \frac{\partial \tau_{\mathrm{yx}}}{\partial \mathrm{x}}+\frac{\partial \sigma_{\mathrm{y}}}{\partial \mathrm{y}}=0, \\
\nabla^{2}\left(\sigma_{x}+\sigma_{y}\right)=\nabla^{2}\left(2 \cdot \sigma_{0}\right)=0
\end{gathered}
$$

граничные условия в напряжениях:

$$
\tau_{n}=-\frac{\sigma_{x}-\sigma_{y}}{2} \cdot \sin 2 \varphi+\tau_{x y} \cdot \cos 2 \varphi,
$$


имеем два уравнения равновесия также уравнение неразрывности деформаций в напряжениях [11], где $\sigma_{0}$ - среднее нормальное напряжение или гидростатическое давление.

В постановке задачи теории упругости используются уравнения (5) и граничные условия (6), которые справедливы для плосконапряженного и плоскодеформированного состояний [12].

Упростим выражение (6), принимая:

$$
\tau_{x y}=T_{i} \cdot \sin (A \Phi), \quad \sigma_{x}-\sigma_{y}=2 \cdot T_{i} \cdot \cos (A \Phi) .
$$

Граничные условия запишутся в виде:

$$
\tau_{n}=-T_{i} \cdot \sin (A \Phi-2 \varphi),
$$

где $T_{i}=T_{i}(x, y)$ - функция координат очага деформации, совпадающая по функциональному назначению с интенсивностью касательных напряжений;

$A$ - постоянный коэффициент, определяющий упругое состояние деформируемой среды;

$\Phi-$ функция координат, характеризующая контактные касательные напряжения, одна из вводимых в рассмотрение аргумент функций.

Второе выражение (7) следует подтвердить решением задачи и тем самым замкнуть его.

В монографии [13] предлагается, в случае линейности дифференциальных уравнений (5), использовать фундаментальную подстановку. В данном случае не следует ограничивать показатель экспоненты линейной функцией, как это рекомендуется в [13], ее следует "отпустить" и получить выражение или условия существования решения задачи. Принимаем:

$$
T_{i}=C_{\sigma} \cdot \exp ( \pm \theta)=C_{\sigma} \cdot[\operatorname{ch}(\theta) \pm \operatorname{sh}(\theta)] .
$$

Используя (7-9), имеем:

$$
\tau_{x y}=C_{\sigma} \cdot \exp (\theta) \cdot \sin (A \Phi), \quad \sigma_{x}-\sigma_{y}=2 \cdot C_{\sigma} \cdot \exp (\theta) \cdot \cos (A \Phi) .
$$

Следует добавить, что показатель экспоненты $\theta$ является неизвестной зависимостью и представляет собой вторую аргумент функцию. Используя подходы решения задач теории упругости при помощи функций комплексных переменных [14-15] в сочетании с методом аргумент функций [6], запишем касательное напряжение (10) в виде:

$$
\tau_{x y}=C_{\sigma} \cdot \frac{\exp (\theta+i A \Phi)-\exp (\theta-i A \Phi)}{2 i} .
$$

При такой постановке вопроса появляется возможность определить из уравнений равновесия нормальные напряжения в аналитическом виде. Для этого необходимо подставить выражение для касательных напряжений (11) в уравнения равновесия. Частные производные имеют вид:

$$
\begin{aligned}
& \frac{\partial \tau_{x y}}{\partial y}=C_{\sigma} \cdot \frac{\left(\theta_{y}+i A \Phi_{y}\right) \cdot \exp (\theta+i A \Phi)-\left(\theta_{y}-i A \Phi_{y}\right) \cdot \exp (\theta-i A \Phi)}{2 i} \\
& \frac{\partial \tau_{x y}}{\partial x}=C_{\sigma} \cdot \frac{\left(\theta_{x}+i A \Phi_{x}\right) \cdot \exp (\theta+i A \Phi)-\left(\theta_{x}-i A \Phi_{x}\right) \cdot \exp (\theta-i A \Phi)}{2 i} .
\end{aligned}
$$

После подстановки производных в дифференциальные уравнения равновесия и разделения переменных получим:

$$
d \sigma_{x}=-C_{\sigma} \cdot \frac{\left(\theta_{y}+i A \Phi_{y}\right) \cdot \exp (\theta+i A \Phi)-\left(\theta_{y}-i A \Phi_{y}\right) \cdot \exp (\theta-i A \Phi)}{2 i} \cdot d x
$$




$$
d \sigma_{y}=-C_{\sigma} \cdot \frac{\left(\theta_{x}+i A \Phi_{x}\right) \cdot \exp (\theta+i A \Phi)-\left(\theta_{x}-i A \Phi_{x}\right) \cdot \exp (\theta-i A \Phi)}{2 i} d y
$$

Интегрировать последние выражения не представляется возможным. Однако если осуществить переход от одной переменной интегрирования к другой, используя условие аналитичности стоящих в скобках функций, что имеет место в (1):

$$
\theta_{x}=-A \Phi_{y}, \quad \theta_{y}=A \Phi_{x}
$$

получим:

$$
\begin{gathered}
d \sigma_{x}=-C_{\sigma} \cdot \frac{\left(A \Phi_{x}-i \theta_{x}\right) \cdot \exp (\theta+i A \Phi)-\left(A \Phi_{x}+i \theta_{x}\right) \cdot \exp (\theta-i A \Phi)}{2 i} \cdot d x, \\
d \sigma_{y}=-C_{\sigma} \cdot \frac{\left(-A \Phi_{y}+i \theta_{y}\right) \cdot \exp (\theta+i A \Phi)-\left(-A \Phi_{y}-i \theta_{y}\right) \cdot \exp (\theta-i A \Phi)}{2 i} d y .
\end{gathered}
$$

Как видно, имеем дифференциальные соотношения, связывающиеся одной переменной. Можно показать, что:

$$
\begin{array}{cc}
A \Phi_{x}-i \theta_{x}=\frac{\theta_{x}+i A \Phi_{x}}{i}, & A \Phi_{x}+i \theta_{x}=-\frac{\theta_{x}-i A \Phi_{x}}{i}, \\
-A \Phi_{y}+i \theta_{y}=-\frac{\theta_{y}+i A \Phi_{y}}{i}, & -A \Phi_{y}-i \theta_{y}=\frac{\theta_{y}-i A \Phi_{y}}{i} .
\end{array}
$$

После подстановки полученных выше зависимостей, интегрирования по частям,

$$
\begin{gathered}
\sigma_{x}=C_{\sigma} \cdot \frac{\exp (\theta+i A \Phi)+\exp (\theta-i A \Phi)}{2}+C, \\
\sigma_{y}=-C_{\sigma} \cdot \frac{\exp (\theta+i A \Phi)+\exp (\theta-i A \Phi)}{2}+C .
\end{gathered}
$$

Переходя к вещественным функциям, имеем:

$$
\sigma_{x}=C_{\sigma} \cdot \exp \theta \cdot \cos A \Phi+C, \quad \sigma_{y}=-C_{\sigma} \cdot \exp \theta \cdot \cos A \Phi+C .
$$

Подставляя выражения (12) в разность нормальных напряжений, убеждаемся, что соотношения (10) выполняются. Это подтверждает достоверность используемых граничных условий (6-8). Если интегрированием определялись не напряжения, а девиаторы напряжений:

$$
s_{x}=\sigma_{x}-\sigma_{0}, \quad s_{y}=\sigma_{y}-\sigma_{0},
$$

согласно [3], то:

$$
\sigma_{x}=C_{\sigma} \cdot \exp \theta \cdot \cos A \Phi+\sigma_{0}, \quad \sigma_{y}=-C_{\sigma} \cdot \exp \theta \cdot \cos A \Phi+\sigma_{0} .
$$

Девиаторные составляющие можно записать в виде:

$$
s_{x}=\sigma_{x}-\sigma_{0}-f(y), \quad s_{y}=\sigma_{y}-\sigma_{0}-f(x),
$$

тогда

$$
\begin{gathered}
\sigma_{x}=C_{\sigma} \cdot \exp \theta \cdot \cos A \Phi+\sigma_{0}+f(y), \\
\sigma_{y}=-C_{\sigma} \cdot \exp \theta \cdot \cos A \Phi+\sigma_{0}+f(x),
\end{gathered}
$$


при

$$
\theta_{x}=-A \Phi_{y}, \quad \theta_{y}=A \Phi_{x}, \quad \theta_{x x}+\theta_{y y}=0, A \Phi_{x x}+A \Phi_{y y}=0
$$

Выше приведенные соотношения Коши-Римана аргумент функций полностью замыкают решение задачи и по граничным условиям (8), и уравнениям равновесия (1). Вводимые в рассмотрение неизвестные функции $\theta$ и $A \Phi$ (9-11) определяются уравнениями Лапласа, согласно (15), что вносит достаточную определенность для их нахождения.

Однако задача не завершена, т.к. не определено среднее нормальное напряжения, входящее в (13), (14). В постановке задачи дифференциальное уравнение, определяющее среднее нормальное напряжение, является уравнением неразрывности деформаций. Анализ показывает, что для более полного удовлетворения граничным и очевидным условиям в зоне упругого деформирования подойдет выражение, которое является основой решения для нормальных напряжений (13), (14). В этом случае ставится задача: определить, при каких значениях аргумент функций условие неразрывности (5) будет удовлетворено. Запишем (5) через функцию комплексной переменной:

$$
\nabla^{2}\left(C_{\sigma} \cdot \frac{\exp (\theta+i A \Phi)+\exp (\theta-i A \Phi)}{2}\right)=0 .
$$

Распишем производные по координатам:

$$
\begin{gathered}
\quad \frac{\partial^{2}\left[C_{\sigma} \cdot \frac{\exp (\theta+i A \Phi)+\exp (\theta-i A \Phi)}{2}\right]}{\partial x^{2}}= \\
=C_{\sigma} \frac{\left[\left(\theta_{x x}+i A \Phi_{x x}\right)+\left(\theta_{x}+i A \Phi_{x}\right)^{2}\right] \cdot \exp (\theta+i A \Phi)+}{2} \ldots \\
+\ldots \frac{\left[\left(\theta_{x x}-i A \Phi_{x x}\right)+\left(\theta_{x}-i A \Phi_{x}\right)^{2}\right] \cdot \exp (\theta-i A \Phi)}{2}, \\
=C_{\sigma} \frac{\left[\left(\theta_{y y}+i A \Phi_{y y}\right)+\left(\theta_{y}+i A \Phi_{y}\right)^{2}\right] \cdot \exp (\theta+i A \Phi)+}{2} \ldots \\
+\ldots \frac{\partial^{2}\left[C_{\sigma} \cdot \frac{\exp (\theta+i A \Phi)+\exp (\theta-i A \Phi)}{2}\right]}{2} \\
+\left[\left(\theta_{y y}-i A \Phi_{y y}\right)+\left(\theta_{y}-i A \Phi_{y}\right)^{2}\right] \cdot \exp (\theta-i A \Phi) \\
\quad
\end{gathered}
$$

После подстановки производных в уравнение неразрывности деформаций и сокращений, получим:

$$
\begin{aligned}
& \exp (\theta+i A \Phi) \cdot\left[\left(\theta_{x x}+\theta_{y y}\right)+\left(A \Phi_{x x}+A \Phi_{y y}\right) \cdot i+\left(\theta_{x}+i A \Phi_{x}\right)^{2}+\left(\theta_{y}+i A \Phi_{y}\right)^{2}\right]+ \\
+ & \exp (\theta-i A \Phi) \cdot\left[\left(\theta_{x x}+\theta_{y y}\right)-\left(A \Phi_{x x}+A \Phi_{y y}\right) \cdot i+\left(\theta_{x}-i A \Phi_{x}\right)^{2}+\left(\theta_{y}-i A \Phi_{y}\right)^{2}\right]=0
\end{aligned}
$$


Операторы, стоящие возле экспонент, содержат одинаковые вторые производные по координатам и нелинейности. Если в силу каких-то причин операторы равны нулю, то имеет место тождество. Покажем это. Распишем нелинейности в операторах и перегруппируем их:

$$
\begin{gathered}
\left(\theta_{x}+i A \Phi_{x}\right)^{2}+\left(\theta_{y}+i A \Phi_{y}\right)^{2}= \\
=\left(\theta_{x}+A \Phi_{y}\right) \cdot\left(\theta_{x}-A \Phi_{y}\right)+2 i\left(\theta_{x} \cdot A \Phi_{x}+\theta_{y} \cdot A \Phi_{y}\right)+\left(\theta_{y}+A \Phi_{x}\right) \cdot\left(\theta_{y}-A \Phi_{x}\right) \\
\left(\theta_{x}-i A \Phi_{x}\right)^{2}+\left(\theta_{y}-i A \Phi_{y}\right)^{2}=\left(\theta_{x}+A \Phi_{y}\right) \cdot\left(\theta_{x}-A \Phi_{y}\right)-2 i\left(\theta_{x} \cdot A \Phi_{x}+\theta_{y} \cdot A \Phi_{y}\right)+ \\
+\left(\theta_{y}+A \Phi_{x}\right) \cdot\left(\theta_{y}-A \Phi_{x}\right) .
\end{gathered}
$$

Принимая в произведениях скобок одну равную нулю, уходим от нелинейности, тогда:

$$
\theta_{x}=-A \Phi_{y}, \theta_{y}=A \Phi_{x} .
$$

Данные соотношения представляют собой соотношения Коши-Римана, что имело место при решении дифференциальных уравнений равновесия упругой задачи (5) и решения плоской задачи теории пластичности (1). Подставляя их, автоматически превращается в нуль выражение для обоих операторов:

$$
\theta_{x} \cdot A \Phi_{x}+\theta_{y} \cdot A \Phi_{y}=-A \Phi_{y} \cdot A \Phi_{x}+A \Phi_{x} \cdot A \Phi_{y}=0
$$

После этого уравнение (16) существенно упрощается и принимает вид:

$$
\begin{aligned}
& \exp (\theta+i \mathrm{~A} \Phi) \cdot\left[\left(\theta_{x x}+\theta_{y y}\right)+\left(A \Phi_{x x}+A \Phi_{y y}\right) \cdot i\right]+ \\
+ & \exp (\theta-i \mathrm{~A} \Phi) \cdot\left[\left(\theta_{x x}+\theta_{y y}\right)-\left(A \Phi_{x x}+A \Phi_{y y}\right) \cdot i\right]=0 .
\end{aligned}
$$

Из соотношений Коши-Римана определяем вторые производные, которые показывают, что:

$$
\theta_{x x}+\theta_{y y}=0, A \Phi_{x x}+A \Phi_{y y}=0
$$

Уравнение неразрывности деформаций тождественно удовлетворяется. Следовательно, решением уравнения неразрывности деформаций является:

$$
\sigma_{0}=n \cdot C_{\sigma} \cdot \exp \theta \cdot \cos A \Phi,
$$

при $\theta_{x}=-A \Phi_{y}, \theta_{y}=A \Phi_{x}, \theta_{x x}+\theta_{y y}=0, A \Phi_{x x}+A \Phi_{y y}=0$, где $n-$ любое число.

На решение (17) накладываются те же ограничения, что и для (13-14), при тех же параметрах. Следует подчеркнуть, что решение уравнения неразрывности деформаций допускает присутствия в выражении среднего нормального напряжения одновременно двух экспонент с противоположными знаками аргумент функции $\theta$. Покажем это:

$$
\sigma_{0}=n \cdot C_{\sigma} \cdot \exp (-\theta) \cdot \cos A \Phi=n \cdot C_{\sigma} \cdot \frac{\exp (-\theta+i A \Phi)+\exp (-\theta-i A \Phi)}{2} .
$$

Подставляя в уравнение неразрывности деформаций, имеем:

$$
\begin{aligned}
& \exp (-\theta+i \mathrm{~A} \Phi) \cdot\left[-\left(\theta_{x x}+\theta_{y y}\right)+\left(A \Phi_{x x}+A \Phi_{y y}\right) \cdot i+\left(\theta_{x}-i A \Phi_{x}\right)^{2}+\left(\theta_{y}-i A \Phi_{y}\right)^{2}\right]+ \\
+ & \exp (-\theta-i \mathrm{~A} \Phi) \cdot\left[-\left(\theta_{x x}+\theta_{y y}\right)-\left(A \Phi_{x x}+A \Phi_{y y}\right) i+\left(\theta_{x}+i A \Phi_{x}\right)^{2}+\left(\theta_{y}+i A \Phi_{y}\right)^{2}\right]=0 .
\end{aligned}
$$

Сопоставляя (16) и (18), убеждаемся, что операторы перед экспонентами противоположного знака в сравнении с функцией $\theta$, с точки зрения решения практически не изменились. 
Таким образом, поставлена и решена плоская задача теории упругости, выявлены обобщающие соотношения (15), определяющие условия существования заданного класса решений через инварианты дифференциальных соотношений аргумент функций.

В общем можно записать:

$$
\begin{array}{rr}
\sigma_{x}=C_{\sigma} \cdot \exp \theta \cdot \cos A \Phi+\sigma_{0}, & \sigma_{y}=-C_{\sigma} \cdot \exp \theta \cdot \cos A \Phi+\sigma_{0}, \\
\tau_{x y}=C_{\sigma} \cdot \exp (\theta) \cdot \sin (A \Phi), & \sigma_{0}=n \cdot C_{\sigma} \cdot \exp \theta \cdot \cos A \Phi,
\end{array}
$$

при $\theta_{x}=-A \Phi_{y}, \quad \theta_{y}=A \Phi_{x}, \quad \theta_{x x}+\theta_{y y}=0, A \Phi_{x x}+A \Phi_{y y}=0$.

Анализ показывает, что решение (19) может быть в дальнейшем усилено и представлено в виде:

$$
\begin{gathered}
\sigma_{x}=\exp \theta\left(C_{1} \cos A \Phi-C_{2} \sin A \Phi\right)+\sigma_{0}=[\operatorname{ch}(\theta)+\operatorname{sh}(\theta)]\left(C_{1} \cos A \Phi-C_{2} \sin A \Phi\right)+\sigma_{0} ; \\
\sigma_{y}=-\exp \theta\left(C_{1} \cos A \Phi-C_{2} \sin A \Phi\right)+\sigma_{0}=-[\operatorname{ch}(\theta)+\operatorname{sh}(\theta)]\left(C_{1} \cos A \Phi-C_{2} \sin A \Phi\right)+\sigma_{0} ; \\
\tau_{x y}=\exp (\theta) \cdot\left(C_{1} \sin A \Phi+C_{2} \cos A \Phi\right)=[\operatorname{ch}(\theta)+\operatorname{sh}(\theta)]\left(C_{1} \sin A \Phi+C_{2} \cos A \Phi\right) ; \\
\sigma_{0}= \pm n \cdot \exp \theta\left(C_{1} \cos A \Phi \mp C_{2} \sin A \Phi\right)= \pm n \cdot[\operatorname{ch}(\theta)+\operatorname{sh}(\theta)]\left(C_{1} \cos A \Phi \mp C_{2} \sin A \Phi\right) \\
\text { при } \theta_{x}=-A \Phi_{y}, \theta_{y}=A \Phi_{x}, \theta_{x x}+\theta_{y y}=0, A \Phi_{x x}+A \Phi_{y y}=0 .
\end{gathered}
$$

Если в показателе экспоненты взят знак минус, $\exp (-\theta)$, тогда структура формул (20) несколько изменится:

$$
\begin{gathered}
\sigma_{x}=-\exp (-\theta)\left(C_{1} \cos A \Phi-C_{2} \sin A \Phi\right)+\sigma_{0}=-[\operatorname{ch}(\theta)-\operatorname{sh}(\theta)]\left(C_{1} \cos A \Phi-C_{2} \sin A \Phi\right)+\sigma_{0} ; \\
\sigma_{y}=\exp (-\theta)\left(C_{1} \cos A \Phi-C_{2} \sin A \Phi\right)+\sigma_{0}=[\operatorname{ch}(\theta)-\operatorname{sh}(\theta)]\left(C_{1} \cos A \Phi-C_{2} \sin A \Phi\right)+\sigma_{0} ; \\
\tau_{x y}=\exp (-\theta)\left(C_{1} \sin A \Phi+C_{2} \cos A \Phi\right)=[\operatorname{ch}(\theta)-\operatorname{sh}(\theta)]\left(C_{1} \sin A \Phi+C_{2} \cos A \Phi\right) ; \\
\sigma_{0}= \pm n \cdot \exp (-\theta)\left(C_{1} \cos A \Phi \mp C_{2} \sin A \Phi\right)= \pm n \cdot[\operatorname{ch}(\theta)-\operatorname{sh}(\theta)]\left(C_{1} \cos A \Phi \mp C_{2} \sin A \Phi\right) \\
\text { при } \quad \theta_{x}=A \Phi_{y}, \quad \theta_{y}=-A \Phi_{x}, \quad \theta_{x x}+\theta_{y y}=0, A \Phi_{x x}+A \Phi_{y y}=0 .
\end{gathered}
$$

Как частный вариант, выражения (21) можно рассматривать как функцию напряжений и его можно привлечь для сравнительного анализа. Действительно, бигармоническое уравнение для плоской задачи можно представить:

$$
\nabla^{4} \varphi=\nabla^{2}\left(\nabla^{2} \varphi\right)=0
$$

Как было показано выше:

$$
\nabla^{2}\left(\sigma_{0}\right)=0 \text {. }
$$

Следовательно:

$$
\nabla^{2}\left[\nabla^{2}\left(\sigma_{0}\right)\right]=\nabla^{2}[0]=0
$$

В работе [12] показаны решения плоской задачи при помощи тригонометрических рядов. Функция напряжений $\varphi$ имеет вид:

$$
\varphi=\sin (\alpha x) \cdot\left[C_{3} \cdot \operatorname{ch}(\alpha \cdot y)+C_{4} \cdot \operatorname{sh}(\alpha \cdot y)+C_{5} \cdot y \cdot \operatorname{ch}(\alpha \cdot y)+C_{6} \cdot y \cdot \operatorname{sh}(\alpha \cdot y)\right] .
$$

Приведем выражения (21) и (22) в сопоставимый вид, т. е. $C_{5}=C_{6}=0, \mathrm{~A} \Phi=\alpha x$, $\theta=\alpha y, n=1, C_{1}=0, C_{2}=-1$. Должны быть справедливы соотношения Коши-Римана и уравнения Лапласа, которые были получены настоящим решением: 


$$
\theta_{x}=-A \Phi_{y}, \theta_{y}=A \Phi_{x}, \theta_{x x}+\theta_{y y}=0, A \Phi_{x x}+A \Phi_{y y}=0
$$

Проверим выполнение указанных обобщений. Действительно $\theta_{x}=0, A \Phi_{y}=0$, $\theta_{y}=\alpha, A \Phi_{x}=\alpha$. Тогда соотношения Коши-Римана для известного решения также имеют место $0=-0, \alpha=\alpha$, т. е. полученные в работе [12] функции являются частным решением по отношению к (21). Это следует из того, что функции $\alpha \cdot x$ и $\alpha \cdot y$ являются простейшим решением уравнения Лапласа, которое допускает целый класс гармонических функций в различных сочетаниях. Аргумент функции не обязательно должны быть линейными и могут, зависеть одновременно от нескольких переменных. К примеру, более сложной функцией $A \Phi$ является функция второго порядка:

$$
A \Phi=\alpha \cdot x \cdot y .
$$

Она удовлетворяет уравнению Лапласа и, через соотношение Коши-Римана, определяет вторую аргумент функцию $\theta$, вида:

$$
\theta=-\frac{1}{2} \cdot \alpha \cdot\left(x^{2}-y^{2}\right)
$$

Сопоставляя решения (1), (2) и (20), (21), полученные одним методом (методом аргумент функций), следует отметить, что между ними существует достаточно очевидное соответствие. Используются одинаковые базовые функции, между аргумент функциями определены одни и те же дифференциальные соотношения Коши-Римана, показан одинаковый тип аргумент функций, удовлетворяющих уравнению Лапласа. Принципиальным отличием являются разные граничные условия, обеспечивающие решения упругой и пластической задач механики сплошной среды.

\section{ВЫВОДЫ}

На базе методов аргумент функций и функций комплексной переменной показан новый подход решения плоской задачи теории упругости.

Показано соответствие решений плоской задачи теории упругости и пластичности.

Предложенные аналитические решения механики сплошной среды могут быть использованы при анализе напряженного состояния металла в очаге деформации, находящегося одновременно в двух состояниях упругом и пластичном.

\section{СПИСОК ИСПОЛЬЗОВАННОЙ ЛИТЕРАТУРЫ}

1. Ильюшин А. А. Пластичность / А. А. Ильюшин. - М. : ОГИЗ ГОСТЕХИЗДАТ, 1949. - 376 с.

2. Качанов Л. М. Основы теории пластичности /Л. М. Качанов. - М. : Наука, 1969. - 420 с.

3. Малинин М. М. Прикладная теория пластичности и ползучести / М. М. Малинин. - М. : Машиностроение, 1975. - 398 с.

4. Павлов И. М. Теория прокатки / И. М. Павлов. - М. : Металлургиздат, 1950. - 511 с.

5. Тарновский И. Я. Деформации и усилия при обработке металлов давлением / И. Я. Тарновский, А. А. Поздеев, О. А. Ганаго. - М. : Машгиз, 1953. - 304 с.

6. Чигиринский В. В. Метод решения задач теории пластичности с использованием гармонических функций / В. В.Чигиринский // Изв. вузов. Черная металлургия. - 2009. - № 5. - С. 11-16.

7. Чигиринский В. В. О новых подходах решения задач теории пластичности / В. В. Чигиринский // Обработка материалов давлением : сб. науч. трудов. - Краматорск : ДГМА, 2009. - № 1 (20). - С. 41-49.

8. Chigurinski $V$. V. The study of stressed and deformed metal state under condition of no uniform plastic medium flow / V. V. Chigurinski // Metalurgija. - Zagreb. - 1999. - Vol. 38, Br. 1. - P. 31-37.

9. Chigirinsky V.V. Development of dynamic model of transients in mechanical systems using argumentfunctions / V. Chigirinsky, A. Putnoki // Easten-European Journal of Technologies. Applied mechanics. - 2017. № 3/7(87). - P. 11-21.

10. Производство тонкостенного проката специиального назначения / В. В. Чигиринский, Ю. С. Кресанов, А. Я. Качан и др. - Запорожье, 2014. - 285 c. 
11. Безухов Н. И. Основы теории упругости, пластичности и ползучести / Н. И. Безухов. - 2-е изд., испр. и доп. - М. : Высш. ик., 1968. - 512 c.

12. Никифоров С. Н. Теория упругости и пластичности / С. Н. Никифоров. - М. : ГИЛСИ, 1955. - 284 с.

13. Тихонов А. Н. Уравнения математической физики / А. Н. Тихонов, А. А. Самарский. - М. : Изд-во МГУ, 1999. - $799 c$.

14. Тимошенко С. П. Теория упругости / С. П. Тимошенко. - Л. : ОНТИ, 1934. - 451 с.

15. Мусхелишвили Н. И. Некоторые основные задачи математической теории упругости / Н. И. Мусхелишвили. - М. : Наука, 1966. - 547 с.

\section{REFERENCES}

1. Il'jushin A. A. Plastichnost' / A. A. Il'jushin. - M. : OGIZ GOSTEHIZDAT, 1949. - 376 s.

2. Kachanov L. M. Osnovy teorii plastichnosti / L. M. Kachanov. - M. : Nauka,1969. - 420 s.

3. Malinin M. M. Prikladnaja teorija plastichnosti i polzuchesti / M. M. Malinin. - M. : Mashino-stroenie, 1975. - $398 \mathrm{~s}$.

4. Pavlov I. M. Teorija prokatki / I. M. Pavlov. - M. : Metallurgizdat, 1950. - 511 s.

5. Tarnovskij I. Ja. Deformacii i usilija pri obrabotke metallov davleniem / I. Ja. Tarnovskij, A. A. Pozdeev, O. A. Ganago. - M. : Mashgiz, 1953. - 304 s.

6.Chigirinskij V. V. Metod reshenija zadach teorii plastichnosti s ispol'zovaniem garmonicheskih funkcij / V. V.Chigirinskij // Izv. vuzov. Chernaja metallurgija. - 2009. - № 5. - S. 11-16.

7. Chigirinskij V. V. O novyh podhodah reshenija zadach teorii plastichnosti / V. V. Chigirinskij // Ob-rabotka materialov davleniem : sb. nauch. trudov. - Kramatorsk: DGMA, 2009. - № 1 (20). - S. 41-49.

8. Chigurinski $V . V$. The study of stressed and deformed metal state under condition of no uniform plastic medium flow / V. V. Chigurinski // Metalurgija. - Zagreb. - 1999. - Vol. 38, Br. 1. - P. 31-37.

9. Chigirinsky V.V. Development of dynamic model of transients in mechanical systems using argumentfunctions / V. Chigirinsky, A. Putnoki // Easten-European Journal of Technologies. Applied mechanics. - 2017. № 3/7(87). - P. 11-21.

10. Proizvodstvo tonkostennogo prokata special'nogo naznachenija / V. V. Chigirinskij, Ju. S. Kresa-nov, A. Ja. Kachan i dr. - Zaporozh'e, 2014. - 285 s.

11. Bezuhov N. I. Osnovy teorii uprugosti, plastichnosti i polzuchesti / N. I. Bezuhov. - 2-e izd., ispr. i dop. M. : Vyssh. shk., 1968. - 512 s.

12. Nikiforov S. N. Teorija uprugosti i plastichnosti / S. N. Nikiforov. - M. : GILSI, 1955. - 284 s.

13. Tihonov A. N. Uravnenija matematicheskoj fiziki / A. N. Tihonov, A. A. Samarskij. - M. : Izd-vo MGU, 1999. - 799s.

14. Timoshenko S. P. Teorija uprugosti / S. P. Timoshenko. - L. : ONTI, 1934. - 451 s.

15. Mushelishvili N. I. Nekotorye osnovnye zadachi matematicheskoj teorii uprugosti / N. I. Mushelishvili. - M. : Nauka, 1966. - $547 \mathrm{~s}$.

Чигиринский В. В. - д-р техн. наук, проф., пом. дир. ЧАО «КрКЗ»;

Науменко Е. Г. $\quad$ - ст. преп. НТУ «Днепровская политехника».

ЧАО «КрКЗ» - Частное акционерное общество «Кременчугский колесный завод», г. Кременчуг.

НТУ «Днепровская политехника»-Национальный технический университет «Днепровская политехника», г. Днепр.

E-mail: val.chig1948@gmail.com; elenanaumenko1971@,gmail.com. 